\title{
Cutaneous atypical mycobacteriosis
}

R Sultana ${ }^{1}, \mathbf{M}$ Wahiduzzaman ${ }^{2}$

\begin{abstract}
Mycobacterium chelonae rarely causes skin infection, usually after minor trauma. A 44 year old lady complained of skin rashes on left lateral back above the flank for 6 months. Physical examination showed multiple papulo-pustular eruption and sinus on an erythematous base. Repeated histopathological examination did not give any authentic clue. Ultimately on clinical ground and therapeutic trial, it was diagnosed as rapidly growing atypical mycobacterial (Mycobacterium chelonae) infection.
\end{abstract}

\section{Introduction}

Atypical mycobacteria affect the skin and result in a variety of lesion including ulceration, rheumatoid like nodule, abscess and histopathological features such as granuloma, diffuse histiocytic reaction and panniculitis. The organisms are found in wet soil, house dust, water, dairy products, cold blooded animals, vegetables and human feces. Amongst the atypical mycobacteria M. chelonae/absessus group usually cause subcutaneous abscess and cellulitis. The bacteria are accidentally transmitted by inhalation, ingestion, percutaneous penetration such as skin piercing, C02 laser resurfacing, laser hair removal, mesotherapy, liposuction, catheterization and cause disease in the skin, lung, lymph node, skeletal and GI systems.1,2 These rapidly growing mycobacteria are frequently resistant to standard antimicrobial medication.' Extensive scarch revealed that no such case has been published in our country till now.

\section{Case report}

A 44 year old lady with skin rash in left lower back presented in OPD in Dermatology Department in Khulna Medical College Hospital, Khulna. Her lesions first appeared in January 2016 as multiple papule which rapidly evolve into pustule on an erythematous base. She had been treated with repeated courses of multiple antibiotic for 3 months but she could not recollect the name of the drugs and the result was unsatisfactory and worsening. Ultimately, she attended the department of Dermatology and Venereology in OPD.

On examination, we have found multiple, discrete, papulo pustular lesion on an erythematous base with discharging sinus on left lower back above the flank (fig. 1). These lesions are slightly itchy, some are scarring without involving other area. There is no mucosal lesion. Again there was no history of trauma, injection or any other penetrating injury or catheterization. On query, she did not give any history of fever, cough, breathlessness, dysuria. On clinical ground, differential diagnosis of deep mycosis, actinomycosis, papulo necrotic tuberculosis, scrofuloderma and atypical mycobacterial infection i,e M. chelonae infection was made.

Lab investigation for relevant test such as CBC, CXR, urine routine and MT was within normal limit. On the basis of histopathological report, which was claimed to be papulonecrotic tuberculid, patient was treated with anti tubercular chemotherapy (CAT-1) for 6 months under supervision but there was no improvement rather than worsening the sign and symptom with 'involving adjacent area. After 7 months patient revisited to Skin OPD and at that time doxycycline $100 \mathrm{mg}$ twice daily was prescribed for 1 month and after that there was a little improvement.

Due to absence of notable improvement, rebiopsy was taken for histopathological examination. This time histopathology report showed chronic dermatitis with superadded infection. Flucloxacillin $500 \mathrm{mg} 4$ times daily was prescribed along with potassium permanganate wash and nebanol powder (neomycin, bacitracin \& zinc sulfate) which did not show marked improvement.

On 1st January 2017, according to differential diagnosis, M. chelonae infection was considered and clarithromycin 500mg bid with doxycyclie 100 mg bid was given for 6 months. Patient showed 
moderate resolution of lesion within 2 months after starting treatment (Fig-2). Five and a half months later her lesion was completely clear, leaving punched out scar (fig 3).

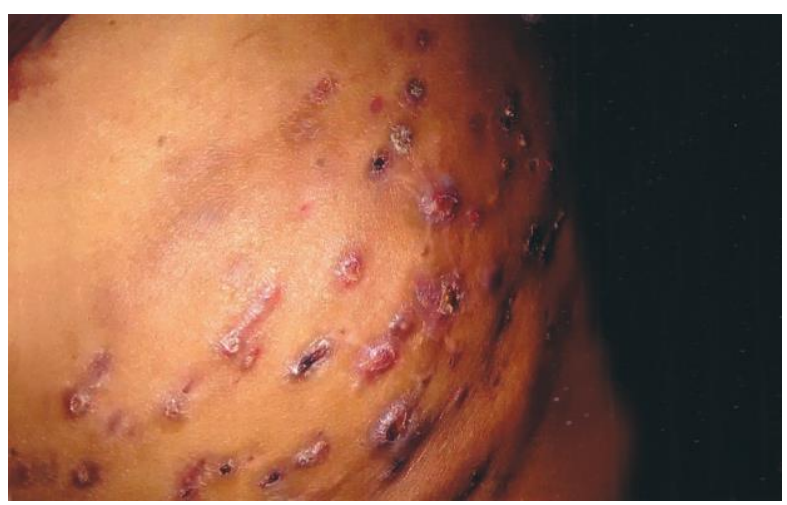

Fig. 1: Papulo-pustule with discharging sinus (fist visit)

\section{Discussion}

This case demonstrates the possibility of infection with the low pathogenic facultative group IV atypical mycobacteria, especially $\mathrm{M}$. chelonae. The clinical presentation of skin infection caused by atypical mycobacteria varies and often leads to the delay in diagnosis which is similar in respect to our reported case. ${ }^{3}$ Reports have shown that post injection abscess with $\mathrm{M}$. chelonae but there is no history of penetrating injury in our case.

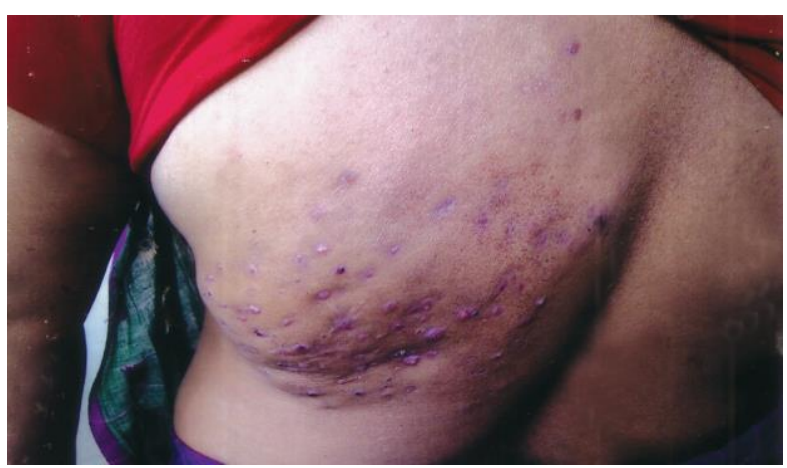

Fig. 2: Two months after treatment

Cutaneous infection with rapidly growing mycobacteria can manifest in a variety of way including ulceration, abscess, draining sinuses or nodules. ${ }^{4}$ Clinical presentation of our case confirms this published literature. This case represents an example of diagnostic dilemma of atypical mycobacterial infection because histopathology is often inconclusive. ${ }^{5}$ Although PCR and culture give the highest diagnostic yield, are rarely available in our country and this is the loop hole and pitfall in this study.

A high index of suspicion is needed when dealing with the case of non specific skin infection which show no clinical response to standard treatment. In some patient, clinical diagnosis must be made and empiric therapy is given. Regarding treatment, it is reported that oral clarithromycin and doxycycline give excellent result leaving atrophic scar. ${ }^{7}$ We found no reported case of $\mathrm{M}$. chelonae infection in our country and very few cases are published worldwide. Our case will be an addition to a few reported cases.

Cutaneous atypical mycobacteria is a rare disease, always representing a challenge for dermatologist. In evaluating the case of mycobacteriosis one should be vigilant that there may be $\mathrm{M}$. chelonae infection and it can be performed by good knowledge about clinical features of this disease.

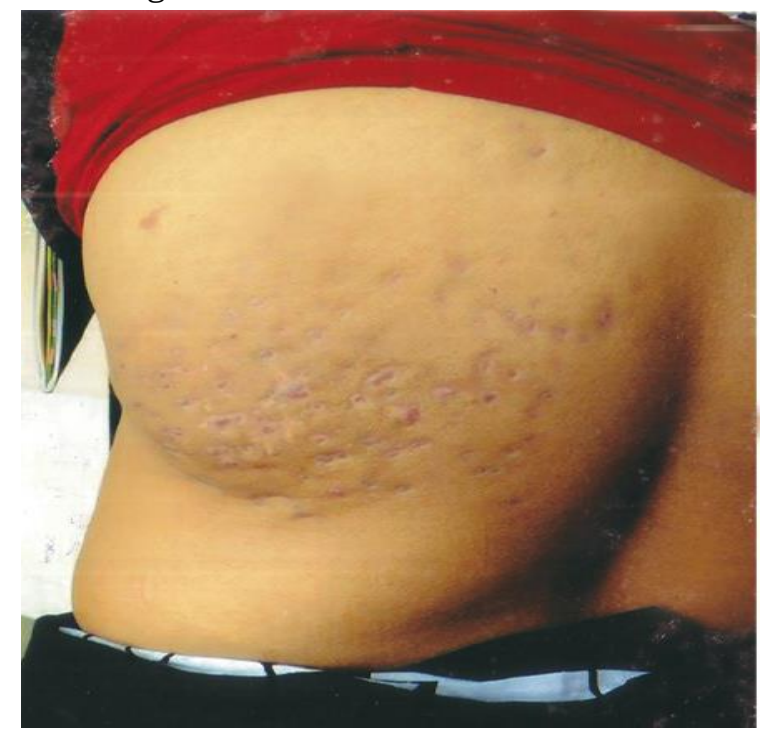

Fig. 3: Healed with atrophic scar

\section{Reference}

1. Willison DJ, Timothy GB, Drik NW, Isac NW. Andrews Disease of the Skin, 12th ed. Philadelphia: Elsevier; 2016. p 326-329.

2. Ho MH, Ho CK, Chong LY. Atypical mycobacterial cutaneous 'infections in Hong Kong: 10 year retrospective study. Hong Kong Med J 2006; 12:2126.

3. Prignano F, Fabroni C, Lohi T. Atypical mycobacteria. InJon C, Hall BJH ed. Skin infection: Cambridge University Press; 2009; 88-91.

4. Lee SH, Kim Ky, Hong SP, Kim MJ, Yang MH and Seou JT. A mycobacterium chelonae sub Sp. Abscess wound infection after percutaneous endoscopic gastrostomy. Korean J Med. 1997; 53: 842-846.

5. Bartralot R, Pujol RM, Garcia Patos V et al. Cutaneous infections due to nontuberculous mycobacteria: histopathological review of 28 cases. Comparative study between lesions observed in immunosuppressed patients and normal hosts. J Cutan. Pathol.2000; 27: 124-129.

6. Young $\mathrm{H}, \mathrm{Ga}$ YL, Wonserk K, Kee JK. A case of skin and soft tissue infection caused by M. Absessus. Am. Dermatol. 2009; 21: 84-87.

7. Barrow T, Legiawati L, Yusharyahya SN, Sularsito SA and Wihadi 1. Atypical mycobacterial infection mimicking carbuncle in an elderly patient. J Gen Pro DVL. 2015; 1: 33-35. 